\title{
Optical coherence tomography angiography (OCTA) applications in ocular oncology
}

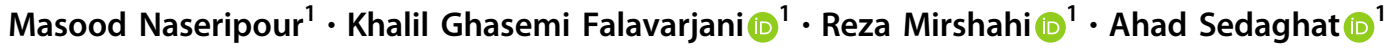

Received: 11 October 2019 / Revised: 4 February 2020 / Accepted: 10 February 2020 / Published online: 3 March 2020

(c) The Author(s), under exclusive licence to The Royal College of Ophthalmologists 2020

\begin{abstract}
Optical coherence tomography angiography (OCTA) is a revolutionary method in the visualization of the vascular system in different retinal and choroidal layers. During the last 4 years since the commercial availability of different OCTA devices, attempts have been made to utilize this technology in various aspects of ocular oncology from the differentiation of benign and malignant lesions to assisting in evaluation of post-treatment complications, such as radiation retinopathy. However, current OCTA technology is restricted by various artefacts and inherent limitations, some of which are more pronounced in the presence of elevated tumoural lesions. Imminent advancements in OCTA systems and image acquisition processes promise a great potential for application of OCTA in ocular oncology.
\end{abstract}

\section{Methodology}

Different combinations of search phrases, including "optical coherence tomography angiography (OCTA)", "Choroidal tumours", "ocular oncology", "uveal melanoma", "Choroidal melanoma", "choroidal nevus", "melanocytoma", "choroidal haemangioma", "choroidal osteoma", "choroidal metastasis", "radiation retinopathy" and "iris" were used for searching PubMed, Scopus and Google Scholar in June 2019 to retrieve articles from 2014. The abstract of the articles was reviewed regarding relevancy to the topic, and the full text of related papers was retrieved for further analysis. Review of non-English material was limited to the available English abstract. A secondary literature search was conducted in September 2019 before the submission.

OCTA is a novel, non-invasive and revolutionary modality in ocular vascular imaging [1]. In contrast to the conventional two-dimensional approaches, such as fluorescein angiography (FA) and indocyanine green (ICG) angiography, OCTA provides depth-resolved volumetric

Ahad Sedaghat

sedaghat.a@iums.ac.ir

1 Eye Research Center, The Five Senses Institute, Rassoul Akram Hospital, Iran University of Medical Sciences, Tehran, Iran information about the microvessels of the choroid and retina without the need for a contrast media [2, 3].

OCTA is an excellent choice to visualize choroidal vasculature, especially in situations in which serial imaging could be beneficial in follow-up and management of the disease, considering the fact that other options, such as FA and ICGA, are time consuming and invasive that might be inconvenient for the patient [3]. In addition, the $3 \mathrm{D}$ data, made available by the OCTA, help with more accurate localization of the abnormal vessels, and provide the clinician with the closer inspection of the deep retinal vasculature [4]. Several studies have shown the ability of the OCTA imaging in depicting vascular alterations in different retinal, choroidal and optic nerve diseases [5-9].

Traditionally, the diagnosis of posterior segment tumours relies on the clinical presentation, ultrasonography and dyebased angiographies. However, differentiation of the true nature of the mass may sometimes pose significant challenges for the physicians. Intrinsic tumoural vasculature may help to differentiate the intraocular tumours [10]. The treatment process may affect the vasculature of the tumour and the surrounding tissue that could be appreciated in detail with OCTA. During the last 4 years, a considerable amount of studies reported OCTA applications to describe different characteristics of ocular tumours. The aim of this study is to review the published articles on the role of the OCTA in intraocular tumours. 
Fig. 1 Choroidal nevus. a Colour fundus photograph of the left eye showing a flat, pigmented and well-demarcated subfoveal nevus. b OCTA B-scan and en face image of choriocapillaris slab show normal vascular flux over the lesion with lack of significant intrinsic vasculature within the nevus.
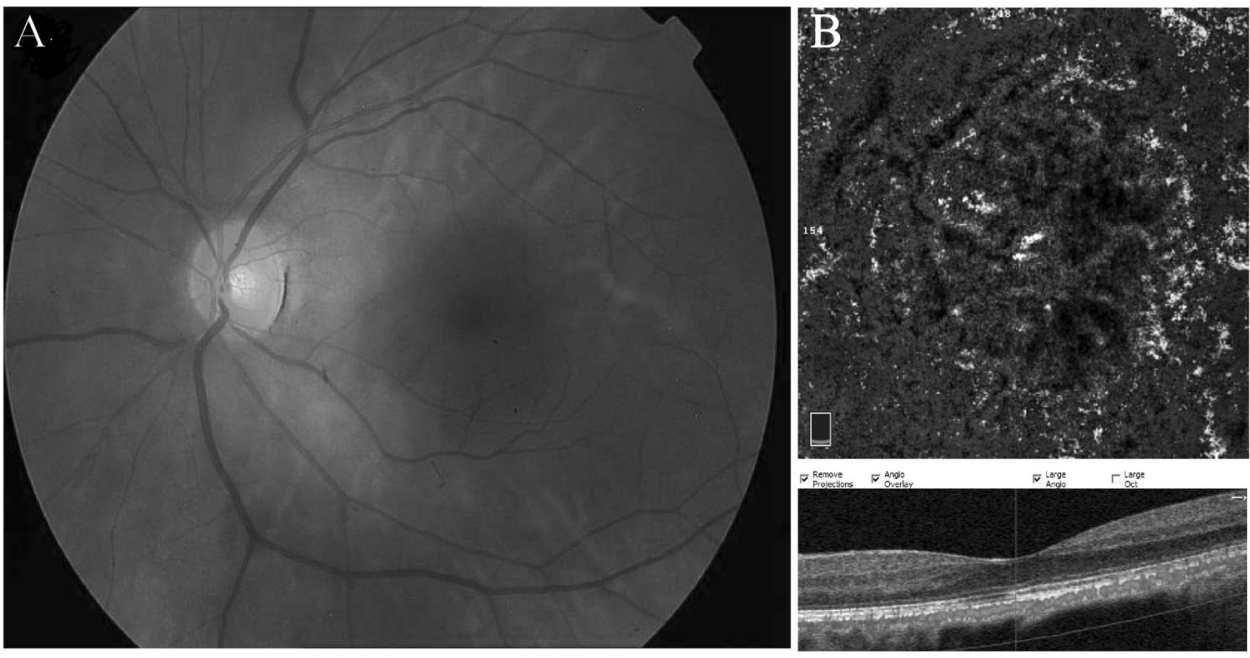

\section{Melanocytic lesions}

Choroidal nevus is a relatively common tumour, particularly in Caucasian individuals, with an estimated prevalence of $0.2-30 \%$ in different reports [11]. Choroidal malignant melanoma (CHMM) is the most common primary intraocular malignancy [12]. It has been shown that some cases of CHMM develop from the pre-existing nevi and others do not $[13,14]$. Melanocytoma is another benign entity in this group, and is usually located juxtapapillary [15].

\section{Diagnosis}

Malignant melanoma is considered to be a sight- and lifethreatening tumour, and based on the results of the Collaborative Ocular Melanoma Study, detection of CHMM in early stages with small dimensions yields a better outcome in terms of both survival and anatomical prognosis [16].

En face OCTA has been shown to have an acceptable reliability in the determination of longitudinal and perpendicular dimensions comparing with the fundus photo with good intra- and inter-rater consistency [17]. In a study of 71 eyes, the visibility of nevus in en face OCTA was $79.5 \%$. Moreover, the thickness greater than $120 \mu \mathrm{m}$ was associated with darker masses in en face OCTA, which could be interpreted as a prognostic factor [18] (Fig. 1).

Clinically, small choroidal melanoma can be distinguished from choroidal nevus based on the tumour thickness, presence of subretinal fluid, orange pigment and visual symptoms in addition to the peripapillary location of the tumour, which are all in favour of malignant melanoma [13]. Acoustic hollowness in ultrasonography and the absence of both halo and drusen are other supplementary findings [13]. However, sometimes a diagnostic challenge appears in differentiating small choroidal melanoma from a choroidal nevus. There is no single modality to successfully distinguish these two entities from each other in all clinical occasions, and multimodal imaging is more effectual [19]. It is hypothesized that malignant transformation of an existing nevus is accompanied by the formation of new and aberrant vessels. Apart from the transformation, complex vascular networks are also usually seen in de novo cases of CHMM [20]. Previously, an effort was made to demonstrate such intrinsic vascularity in some cases in conventional angiography, which was entitled as "double circulation" [21, 22]. It seems rational that OCTA could be helpful in visualization of the vascular loops and networks in CHMM. Maloca et al. [23] implemented an experimental method in speckle noisefree 1050-nm-swept-source OCT for detection of choroidal vessels in small choroidal nevi. Although due to technical issues, the angiography was limited to Haller's and Sattler's layers, their study showed that choroidal vasculature was transformed in the majority of cases. Central signal voids were also noted in most cases in the area of choroidal tumour, probably due to blockage and/or absorption by the heavy pigmented lesion, or the small size of the tumour's vascular network. Radial vessels at the periphery of the tumour were another notable finding, and their calibre was related to the tumour size. In the case of a very small nevus, the choroidal vasculature was completely normal [23].

Further efforts have been made to find differentiating features in OCTA of choroidal melanoma in comparison with choroidal nevus. Toledo et al. reported qualitative assessment of melanocytic lesions, including choroidal nevus with different risk factors for malignancy and frank CHMM, and found that hyperreflective encompassing rings and the presence of intrinsic hyporeflective plexus are indicative of malignant melanocytic lesions [24]. However, not all choroidal melanomas exhibited these features, or were they unique to malignant lesions (Fig. 2).

Ghassemi et al. attempted to differentiate posterior pole choroidal nevus from small choroidal melanoma up to the 
Fig. 2 Choroidal melanoma. a Fundus photo of the left eye showing an elevated, pigmented lesion in the macula with dispersed orange pigment and subretinal fluid at the inferior margin. b OCTA B-scan and the corresponding en face image demonstrate high vascular flux and the overlaying SRF and pigment epithelial detachment associated with outer retina layer disorganization in the corresponding OCT.
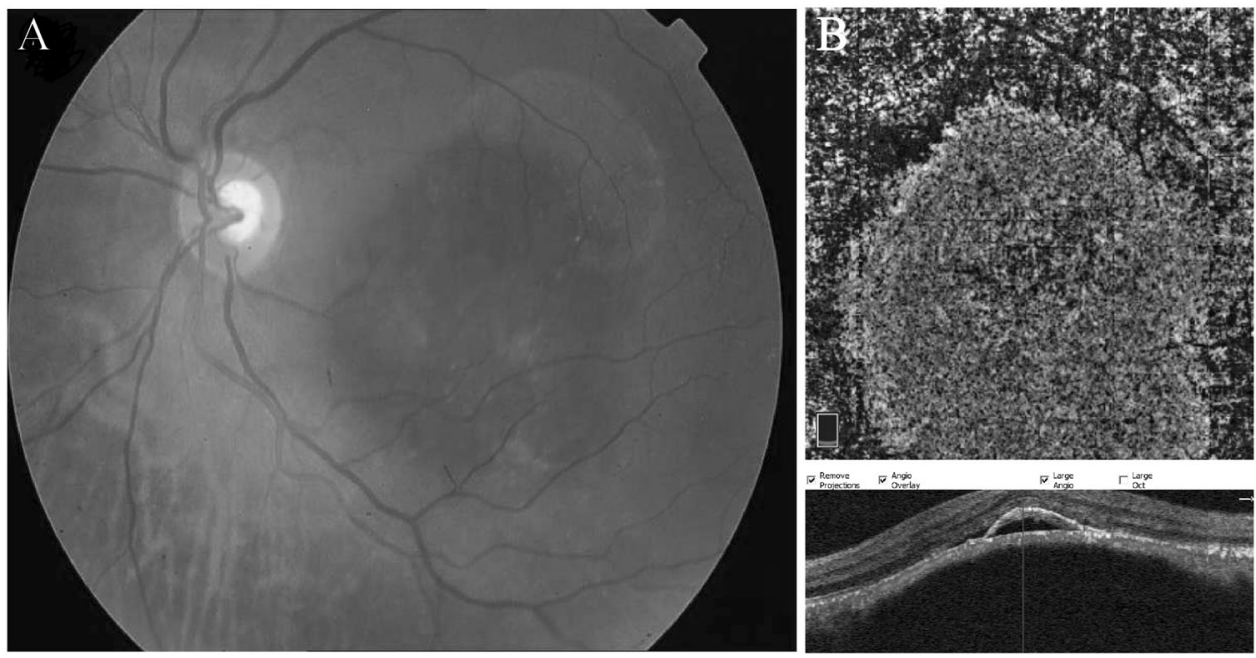

thickness of $3.5 \mathrm{~mm}$, using OCTA. Both melanoma and nevus were identified as hypovascular masses in OCTA imaging, probably due to signal attenuation by the pigment. The concept of surface microvasculature (SMV) was introduced by the authors as a quantitative indicator to distinguish the two entities. The SMV measurement was conducted on the choroidal capillary layer overlying the choroidal mass that was diminished compared with the corresponding surface to the fellow eye in cases of choroidal melanoma, and was intact in nevi. Peripheral feeding vessels were also more prominent in malignant lesions [25].

Similarly, Garcia-Arumi Fuste et al. performed a comparative study on 18 choroidal nevi and 18 choroidal melanomas. They reported that the irregular contour of mass encompassed a hyporeflective area in choriocapillarisoverlying melanoma cases as opposed to hyperreflective choriocapillaris with irregular margins in choroidal nevus. In addition, in $45 \%$ of melanoma patients, OCTA was able to disclose abnormal vasculature, such as loops and thick vessels within the tumour [26].

Cennamo et al. interpreted the OCTA images of different choroidal tumours. Melanocytic lesions in their study included 40 choroidal melanomas, 60 choroidal nevi and 2 optic disc melanocytomas. They reported compression of choroidal vasculature in all melanocytic tumours, the absence of blood flow within nevi and melanocytoma and enhanced visualization of intrinsic vasculature of choroidal melanoma with a thickness of less than $3 \mathrm{~mm}$ [27].

The last two studies were limited by the thickness and location of the tumour, the presence of subretinal fluid and the patients' cooperation. By the advent of the new sweptsource OCTA imaging systems, these barriers are overcome to some extent [3]. Pellegrini and Staurenghi performed swept-source OCTA on a single case of choroidal melanoma with a thickness of $5.9 \mathrm{~mm}$ in the nasal periphery of the fundus, and demonstrated that the intrinsic vessels of the tumour can be disclosed in great detail [28]. The same authors and their colleagues published another paper in 2018 on swept-source imaging of 22 melanoma cases located from macular to the ora serrata and having a thickness of $1.5-8.9 \mathrm{~mm}$. The visualization of the tumours' vasculature was successfully done in all cases by manual adjustment of the segmentation slabs. However, the OCTA of the whole eye was limited in the areas of significant exudative retinal detachment [29]. The "spider web" pattern of intrinsic vasculature in cases of choroidal melanoma, in contrast with the absence of significant vessels in nevus, is suggested to be helpful in the differentiation of these two tumours [30].

\section{Effects on retinal and choroidal structures}

In addition to disclosure of the vasculature within the tumours, OCTA can be helpful in recognition of retinal vasculature alterations caused by the choroidal tumour itself or its secondary complications (e.g., subretinal fluid).

It is shown that relatively flat nevi do not alter the outer retinal vessels in OCTA; however, errors in automatic segmentation of retinal layers disturb the visualization of the vasculature in the outer retina in thicker tumours [18].

$\mathrm{Li}$ et al. demonstrated that there is enlargement of the deep foveal avascular zone (FAZ) and reduction in superficial and deep capillary vascular density (VD) in untreated choroidal melanoma cases, irrelevant of the tumour location and the distance to the fovea. All the mentioned parameters were reversibly correlated with the presence of subretinal fluid. The tumours' dimensions negatively affected the superficial and deep capillary plexus. The proposed hypothesis for these observations is secretion of proinflammatory cytokines, such as vascular endothelium growth factor and subsequent parafoveal ischaemia, or less probably direct tumour invasion to the outer retina [31]. There is concern regarding accurate measurement of the 


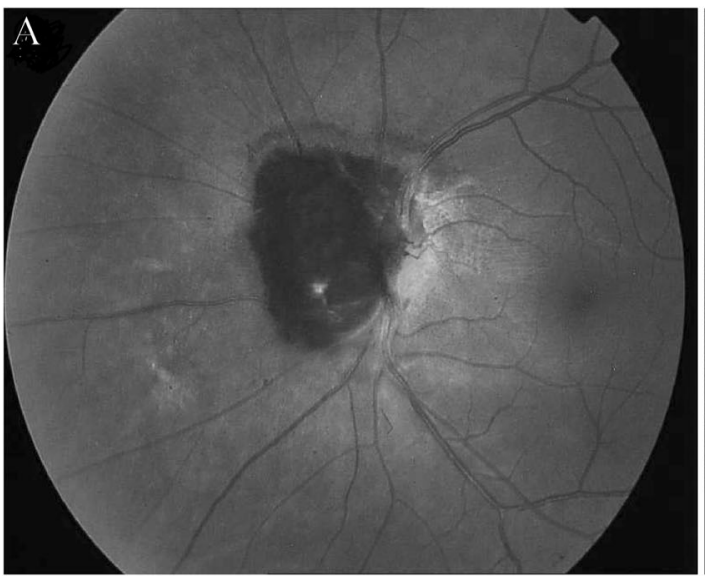

Fig. 3 Optic disc melanocytoma. a Jet-black pigmented lesion overhanging on the nasal part of the disc. b After manual correction of segmentation error, the en face OCTA montage image demonstrates

deep FAZ by the OCTA, especially in areas of intraretinal and subretinal fluid, and increased thickness of the retina. In addition, projection artefacts in retinal pigment epithelium and choroidal layers are more pronounced in the presence of subretinal fluid.

In another study with the use of automated measurements, the macular features of choroidal nevi and melanoma were compared against each other. In choroidal nevi, the FAZ and VD parameters were intact in comparison with the healthy fellow eye, except for a significant reduction of deep VD in tumours with three or more risk factors for growth. On the other hand, superficial and deep VD was diminished in eyes with choroidal melanoma in both macular and extramacular location of the malignant tumour. However, the changes in deep FAZ were observed only in macular melanomas [32]. The difference in alteration of deep FAZ based on the location of the tumour was probably due to the homogeneous distribution of the masses in the fundus in contrast to the macular location of the tumours.

Another application of OCTA is assistance in the diagnosis of choroidal neovascularization (CNV) associated with choroidal nevi. Cicinelli et al. discussed a case of polypoidal with a concomitant peripapillary nevus. The OCTA en face imaging revealed the irregular branching of the new vessels and the hyporeflective areas corresponding to hyalinized polyps with the dimensions of the lesion appearing larger in OCTA than FA and ICGA [33]. A larger retrospective case series by Pellegrini et al. also illustrated that the sensitivity of OCTA in the disclosure of CNV associated with nevus is more than FA and ICG imaging (100\% vs. $90 \%$ and $83 \%$, respectively) [34]. Recently, it has been proposed that avascular zones in OCTA images of optic nerve head in eyes with peripapillary melanocytoma correlate with the location of the visual field defect, indicating that these tumours may disturb the circulation of the optic disc [35] (Fig. 3).

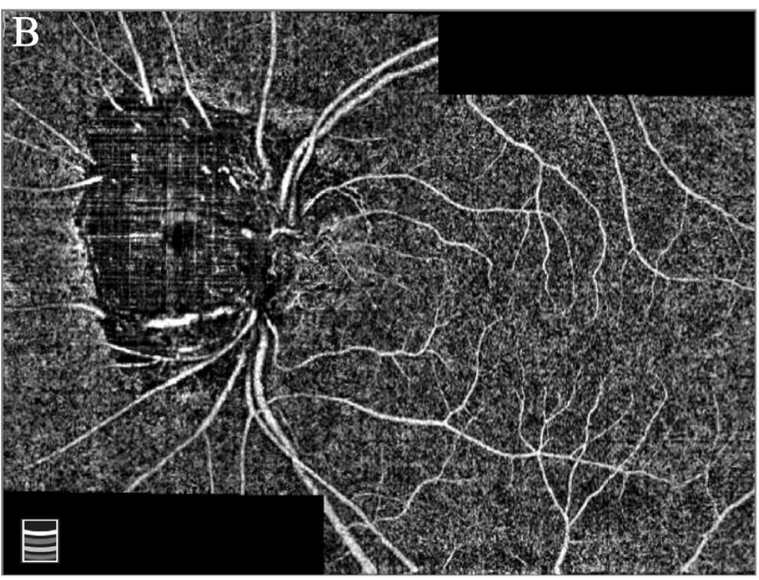

areas of signal void and heterogeneously distributed sparse intrinsic vasculature within the tumour possibly due to the masking effect of pigments.

\section{Post-treatment changes of intrinsic tumour vasculature}

Currently, the mainstay of treatment of choroidal melanoma is radiotherapy, most commonly brachytherapy or proton beam [36]. Considering the high nutritional need of growing malignant tumour, investigation of intrinsic vasculature can be a potential indicator of response to treatment or possibly can be beneficial in highlighting the areas in risk of future growth. Cennamo et al. demonstrated that in comparison with pre-treatment measurements, there is a significant reduction in vessel content and blood flow of the tumours within 1 year after ruthenium-106 plaque brachytherapy [37] (Fig. 4).

In our experience, photodynamic therapy (PDT) and transpupillary thermotherapy (TTT) for treatment of small melanoma have similar effects and result in significant dropout in central tumour vasculature and the overlying choriocapillaris (Fig. 5).

\section{Post-treatment complications}

Radiation retinopathy (RR) is the delayed and major complication of globe conservation therapy by brachytherapy and proton radiation. Prominent involvement of retinal vasculature makes this entity a perfect candidate for evaluation by OCTA. Veverka et al. [38] proposed a grading system based on multimodal imaging including OCTA that was comparable with Horgan modification [39] of the Finger grading system and even more comprehensive. It was shown that OCTA is capable of displaying FAZ enlargement and capillary dropout in RR before the appearance of wellknown signs in ophthalmoscopy or spectral domain OCT imaging. However, as discussed in that article the ability of OCTA to accurately visualize retinal vessels is impaired by the presence of severe oedema. 


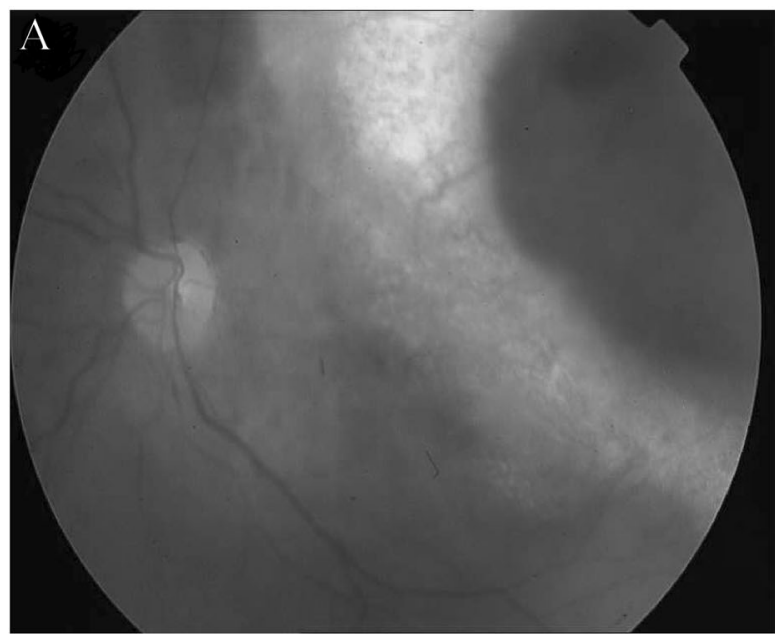

Fig. 4 Choroidal melanoma following Ru-106 plaque radiotherapy. a Colour fundus photograph of the left eye showing a good chorioretinal scar around the pigmented and well-demarcated mass 1

OCTA can also be helpful in the prediction of visual acuity in patients with radiation maculopathy. In a multivariate analysis, Matet et al. reported that the visual acuity was correlated with FAZ size, deep VD and disorganization of deep capillary plexus. There was concern that the measurement of deep VD was confounded by the masking effect of the overlying oedema, but the results were reconfirmed after including the macular thickness in the analysis [40].

Shields et al. reported OCTA images of a combination of cases with and without clinically evident RR following I125 plaque therapy. There was a significant capillary dropout in superficial and deep plexus, in addition to a decrease in VD of choriocapillaris at the tumours' margins. The enlargement of FAZ and a universal decrease in parafoveal VD were other prominent findings [41].

In a study of 112 irradiated eyes without clinical or OCT evidence of RR, 17 months after brachytherapy, OCTA revealed a decrease in the density of parafoveal superficial and deep capillary plexus in spite of the normal FAZ size compared with the healthy fellow eye. It was concluded that the parafoveal ischaemia evident by the OCTA could be a harbinger of RR [42]. However, the measurements in these last three studies were based on a customized method developed by the authors since the articles were published before the recent developments in built-in standard software for the measurement of capillary density.

Cennamo et al. also showed that the VD is diminished following ruthenium-106 plaque therapy at superficial plexus [37]. No effective method was discussed in their study for the reduction of projection artefacts that might influence the density measurements.

The same clinical picture was reported by Sellam et al. who performed OCTA in proton beam-treated eyes
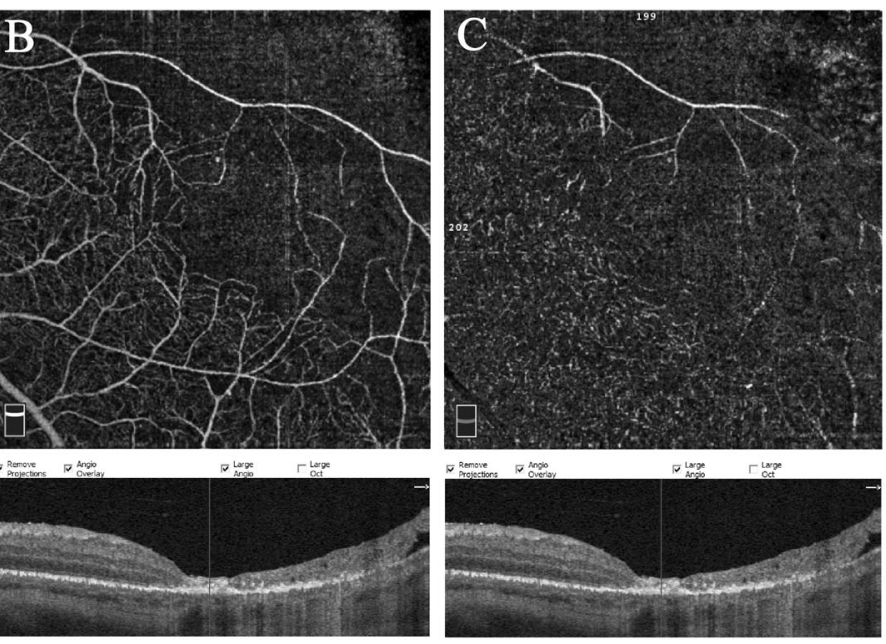

year after brachytherapy. OCTA scan rarefaction of the capillary plexus, both at superficial (b) and deep (c) layers with enlargement and loss of boundary of the foveal avascular zone, is evident.

36 months after treatment. In their study, in order to eliminate segmentation and projection artefacts the measurement of VD was conducted on the whole retina. Imaging abnormality was reported in all cases, and a significant reduction in VD was disclosed in treated eyes in comparison with healthy subjects. The choroid was also affected, and black areas of "signal void" in choriocapillaris, areas of vascular dilation as well as vanishing vessels were displayed in OCTA imaging [43].

Based on the mentioned studies, OCTA can be a valuable tool in early detection of RR. Rarefaction of retinal vasculature both in superficial and deep capillary plexuses in addition to FAZ enlargement precedes future development of radiation maculopathy and decreased vision.

\section{Choroidal haemangioma}

Circumscribed choroidal haemangioma $(\mathrm{CHH})$, a benign hamartomatous vascular lesion, can cause sudden or gradual visual loss in young patients typically aged $20-40$ years [44].

\section{Diagnosis}

The hypervascularity of choroidal haemangioma makes it a perfect candidate for evaluation by OCTA. All studies in the literature are focused on the circumscribed form of choroidal haemangioma, probably due to the presence of normal choroidal vasculature as an internal control for comparison. Although $\mathrm{CHH}$ is a vascular tumour, prominent intrinsic tumour vessels or feeder vessels are not seen ophthalmoscopically. The main finding in OCTA images is irregularly organized intertwined large-calibre vessels surrounded by a circular vascular arcade that acts as the origin of radial 
Fig. 5 Small choroidal melanoma following transpupillary thermotherapy. a Fundus photo disclosing a slightly elevated pigmented lesion underwent transpupillary thermotherapy (TTT) with areas of subretinal fibrosis and RPE changes. b Retinal layer disorganization with significant dropout of tumour vasculature and the overlying

choriocapillaris is evident in the corresponding OCTA scan.
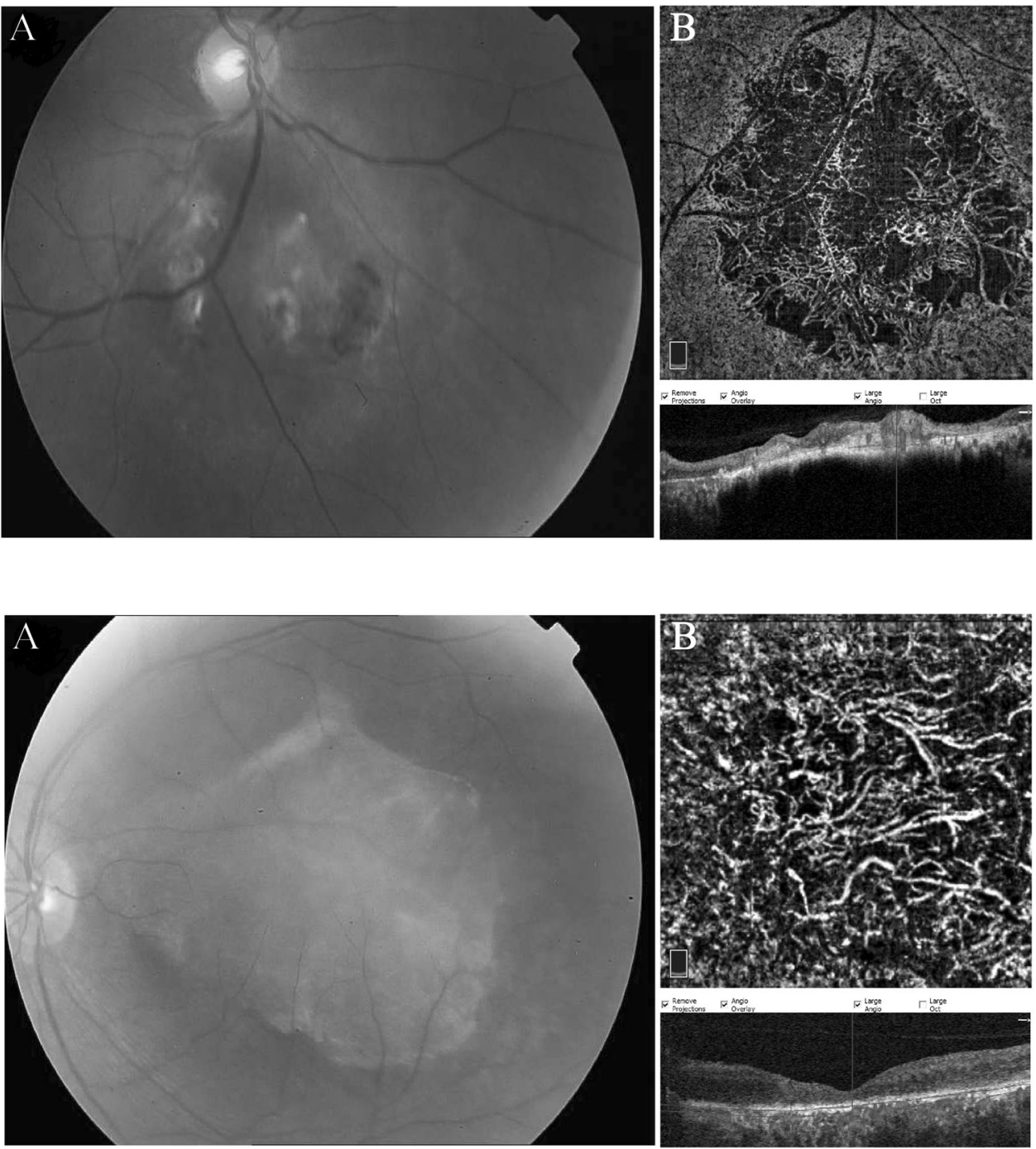

Fig. 6 Circumscribed choroidal haemangioma. a Fundus photo demonstrates an amelanotic submacular mass. b En face choroidal OCTA and Bscan image show visible largecalibre intrinsic vessels with intervening areas of signal void and high vascular flux over the haemangioma. vessels entering the tumour [27, 45, 46]. The signal void formation within the mass might be indicative of shadowing by pigment epithelium or the fringe artefact caused by signal attenuation of deeper layers or very slow blood flow within the vessels [45, 47] (Fig. 6).

\section{Effects on retinal and choroidal structures}

Studies have shown that in case of circumscribed $\mathrm{CHH}$, the abnormality of choroidal vasculature is limited to the zone occupied by the tumour and neighbouring choriocapillaris and other larger vessels appear normal [48].

Sioufi et al. showed that significant changes in deep retinal capillary plexus were exclusive for cases of circumscribed $\mathrm{CH}$ with a previous or concurrent history of subretinal or intraretinal fluid. There was no significant enlargement of FAZ area or reduction in superficial retinal capillary VD in simple or complicated cases of $\mathrm{CH}[48]$.

\section{Post-treatment changes of intrinsic tumour vasculature}

There are many treatment modalities available for management of circumscribed $\mathrm{CH}$ including laser photocoagulation, TTT, PDT, radiotherapy and observation for asymptomatic patients [49]. Chawla et al. reported their preand post-treatment findings on $\mathrm{CHH}$ managed by frequency-doubled $\mathrm{Nd}-\mathrm{YAG}(532 \mathrm{~nm})$ laser. It was observed that Nd-YAG laser does not affect the intrinsic tumour vasculature; however, the irreversible destruction of the overlying choriocapillaris was observed. It is interesting that with the atrophy of choriocapillaris, the visualization of deeper intratumoral vessels is even enhanced [50]. These findings were in favour of Gass's theory that the main goal of laser therapy is approximating the oedematous retina to the underlying tissue in order to improve the absorption of the fluid [51]. Laser photocoagulation does not seem to have any effects on the dimensions of the tumour [50]. 
Fig. 7 Choroidal osteoma. a Fundus photograph represents a circumpapillary amelanotic mass with pseudopod-like projections and areas of ossification. $\mathbf{b}$ OCTA depicts quiescent choroidal neovascularization lesion associated with choroidal osteoma (red arrow) at the outer retinal slab. (Colour figure online).
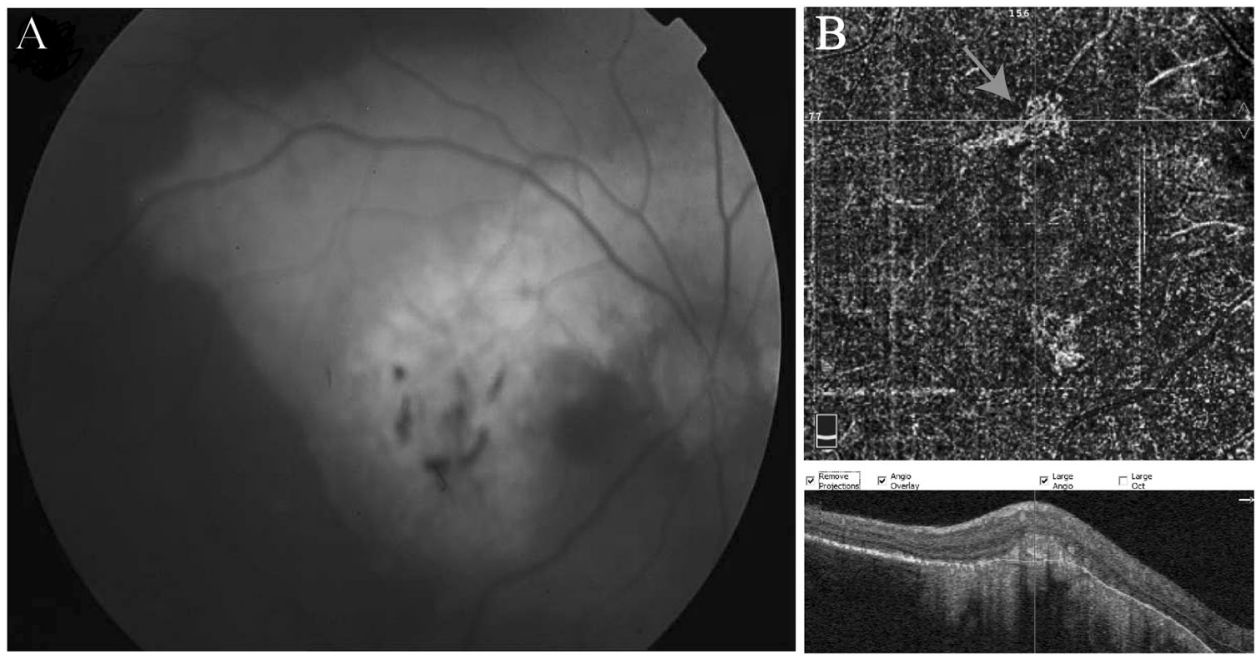

OCTA analysis of post-PDT tumours revealed that as early as 3 days after treatment, the large-calibre intralesional vasculature appears dark probably due to thrombosis and absence of blood flow caused by PDT. However, 7 days later, the vessels recanalize but to a calibre slightly smaller than pre-treatment imaging. The main limitation of this study was the lack of quantitative measurements to accurately illustrate the long-term changes made by PDT [52].

In Cennamo et al.'s study, in spite of the fact that projection artefact was not addressed by the authors, the flow and VD within the tumour demonstrated a significant reduction 1 year after brachytherapy of circumscribed $\mathrm{CHH}$ by ruthenium-106. An actual decrease in the size of the tumour was also observed [53].

\section{Post-treatment complications}

As mentioned earlier, atrophy of choriocapillaris in the region of laser photocoagulation therapy is readily interpreted by OCTA [50]. In addition, the changes associated with RR as a side effect of brachytherapy are discussed in previous sections.

\section{Choroidal osteoma}

Choroidal osteoma is a rare, benign, typically unilateral tumour that usually occurs in healthy young females. The natural history of choroidal osteoma is quite variable with reports of growth, spontaneous resolution, de-ossification, subretinal fluid and development of CNV [54-56]. CNV is the main reason for reduced visual acuity in these patients [57].

\section{Diagnosis}

The diagnosis of choroidal osteoma is clinical and is confirmed by ultrasound sonography [10]. Cennamo et al. reported a fine vascular network in uncomplicated choroidal osteomas, however, the intralesional vascularity had a lesser extent compared with more vascular tumours, such as choroidal haemangioma [27]. There are also reports of signal void in choriocapillaris of the area occupied by choroidal osteoma [58] that may reflect the difference in the ability of different devices in the visualization of deeper vasculature. The major application of OCTA in choroidal osteoma is assistance in the diagnosis of associated $\mathrm{CNV}$, which will be discussed in the next section (Fig. 7).

\section{Effects on retinal and choroidal structures}

The symptoms in choroidal osteoma are usually associated with subretinal fluid caused by the tumour itself or by the CNV [44]. One of the first reports on OCTA in choroidal osteoma accompanied by CNV is published by Azad et al. [59]. The superficial reticular pattern of dilated vessels of CNV was clearly visible by means of OCTA. Also, the additional information about the multiplicity of feeding vessels of the CNV complex helped in treatment planning, rendering laser photocoagulation a less suitable choice [59]. In addition, OCTA is beneficial in disclosing type $2 \mathrm{CNV}$ in outer retina of cases that visualization by FA is obscured by the hemorrhage [60].

\section{Post-treatment changes}

Anti-vascular endothelial growth factor (anti-VEGF) therapy has become the standard of care for the management of 
Fig. 8 Choroidal metastasis. a Fundus photo of a patient with a history of metastatic breast cancer disclosing amelanotic elevated lesion along the inferior arcade with subretinal fluid detaching the fovea. b The en face OCTA at the choroidal level of the corresponding lesion demonstrates an area of signal void (asterisk). The black crescent at the edge of en face images is caused by out-ofregister artefact.
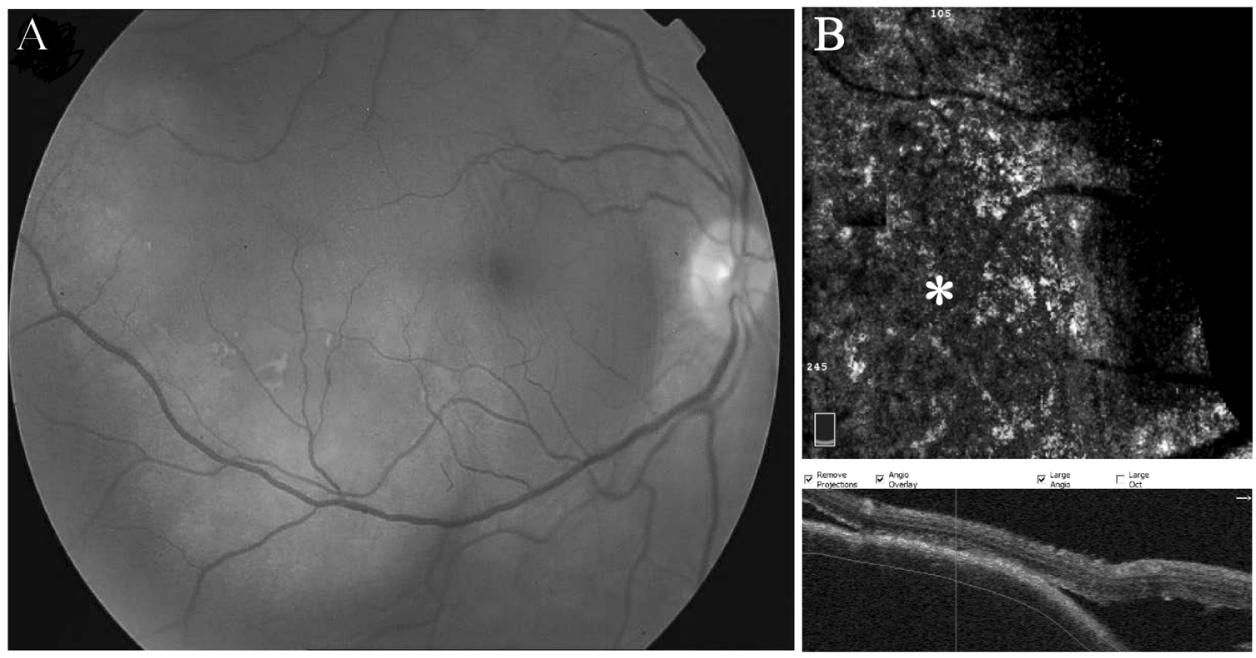

CNV, irrespective of the underlying mechanism. The partial regression of $\mathrm{CNV}$ associated with osteoma following intravitreal injections of anti-VEGF is documented both qualitatively [58] and quantitatively [60, 61]. On the other hand, anti-VEGF does not seem to affect the intrinsic vasculature of the tumour itself [61].

\section{Choroidal metastases}

Choroidal metastasis is the most common cause of intraocular malignancy. In the absence of a reliable history of systemic cancer (e.g., subclinical lung cancer), the conundrum of diagnosis can be a challenge for the ocular oncologist. There are many different modalities useful in the differentiation of metastasis from other choroidal masses [62]. The reports regarding OCTA findings in choroidal metastasis are scarce and most of them counter-intuitively report signal void and hypovascularity in the regions of $\mathrm{CM}$ $[27,62]$. It is hypothesized that lack of blood flow within the metastatic tumour might be due to the shadowing artefact of the overlying retinal oedema and pigment epithelium, or the high velocity of blood flow within the tumour's vasculature (i.e., fringe effect) [62]. However, there are also reports of the observation of thick anastomotic vessels alongside thinner vasculature and hyporeflective avascular regions in OCTA images acquired by "OCT microangiography-complex" algorithm [24] (Fig. 8).

\section{Miscellaneous posterior segment tumours}

In addition to aforementioned tumours, OCTA imaging has been performed in various other intraocular tumours. In a photo essay by Hong and Shields, en face OCT and OCTA were proved useful in the demonstration of the fact that vascular traction and dragging are present in all retinal levels of a case with combined hamartoma of the retina and retinal pigment epithelium [63]. In addition to discernible rarefaction of capillary plexuses and FAZ area, quantitative analysis has also shown that there is a significant reduction of VD in superficial and deep retinal plexus and choriocapillaris in comparison with healthy subjects. The majority of these changes have been reported to be reversible upon vitrectomy of the involved eyes [64].

The diagnosis of juxtapapillary hemangioblastoma can be challenging regarding the lack of feeder or draining vessels and the less probability of association with Von Hippel-Lindau disease than peripheral lesions [65]. It is shown that in OCTA, the exact localization of the tumour to the retina is possible. Hypervascularity of the tumour is also evident by a dense organization of capillary plexus in en face images [66]. Theoretically, visualization of intrinsic vasculature in peripheral tumours can be beneficial in monitoring of treatment response [24].

In another novel application of OCTA in ocular oncology, macular microischaemia was evaluated after intravenous chemotherapy as the mainstay treatment for retinoblastoma. The study showed that in comparison with the control group, there was a significant decrease in VD of deep retinal capillary plexus in both involved and tumourfree eyes 10 years after intravenous chemotherapy. Other measured parameters including visual acuity, central macular thickness, VD of superficial plexus and choriocapillaris and FAZ area were similar to the healthy control group [63]. Although it should be noted that the retrospective nature of the study limits its result-derived conclusions, and perhaps with the likely introduction of handheld OCTA in the future, prospective investigations in young children might be feasible. 


\section{Anterior segment tumours}

Currently, with the manipulation of offset and manual focus changing, OCTA imaging of the anterior segment is possible with some commercially available retinal devices [67]. OCTA has revealed that iris microhemangioma is located at the posterior iris stroma and is fed by normal vasculature of the iris [68].

Iris haemangioma is detected anterior to normal radial vessel and appears as a dilated vessel with U-shaped course from the peripheral iris, forming a tortuous loop near pupil and ending again near the limbal area [69]. The pinpoint vessels of ocular surface squamous neoplasia are also visible in OCTA [67].

In a recent study by Skalet et al. OCTA features of different pigmented lesions of the iris were investigated. The iris freckle and iris pigment epithelial cyst disclosed no intrinsic vascularity. The vascularity of Iris nevus was similar to normal iris stroma. On the other hand, the VD of iris melanoma was significantly higher than the normal iris and benign nevi, and the twisty disorganized vasculature of iris melanoma showed significant regression after brachytherapy [70].

\section{OCTA limitations and future directions}

There are several limitations in current OCTA technology, such as various artefacts, some of which are more significant in elevated and occasionally pigmented ocular lesions [71]. It is reported that at least one type of artefact is present in almost $90 \%$ of OCTA images [72]. Segmentation error is also more pronounced in the presence of increased retinal thickness and elevated masses, which can impede proper quantitative and qualitative assessment of VD in retinal and choroidal pathologies. Therefore, it is of great importance to interpret OCTA images along with other the clinical and imaging findings.

Another concern in eyes with poor visual acuity is the frequent occurrence of different artefacts that affect the signal strength and quality of interpretation. In a recent study by Say et al. in patients with CHMM following plaque radiotherapy, at least one major artefact was evident in $73 \%$ of cases, and loss of focus was the most common one [73]. The reduced image quality-which is not uncommon in eyes with choroidal tumour - can also be detrimental in quantitative and qualitative assessment of OCTA due to various imposed artefacts [74].

It is also important to know that with the increase in the operating wavelength $(1050 \mathrm{~nm}$ of anterior segment OCT vs. $840 \mathrm{~nm}$ of AngioVue OCTA), the penetration of the study light is better in pigmented lesions. However, with darkly coloured iris or heavily pigmented tumour of the iris, the applicability of anterior segment OCTA is limited [70].

With the anticipated future developments of better device algorithms and availability of wide-field SS OCTA better visualization of different ocular tumours is expected. Larger studies with an extensive sample size of different choroidal tumours are needed to elaborate the application of OCTA in ocular oncology further. Artificial intelligence is another high-end technology that can be beneficial in categorizing OCTA findings of choroidal lesions, and improvement of layer segmentation in the presence of an elevated mass disturbing the normal anatomical architecture.

\section{Conclusion}

OCTA imaging is a high-resolution and non-invasive modality that is useful in the disclosure of blood flow, especially in deeper retinal and choroidal layers, which is usually masked by the overlying structures in conventional angiography. This feature of OCTA is helpful in differentiation, treatment planning and follow-up of the intraocular tumours. The swept-source technology of OCTA seems more beneficial in choroidal tumours due to its ability to visualize deeper structures. With optimization of OCTA in imaging of peripheral retina (wide-field OCTA), the applicability of this modality will expand in ocular oncology.

\section{Compliance with ethical standards}

Conflict of interest The authors declare that they have no conflict of interest.

Publisher's note Springer Nature remains neutral with regard to jurisdictional claims in published maps and institutional affiliations.

\section{References}

1. Sambhav K, Grover S, Chalam KV. The application of optical coherence tomography angiography in retinal diseases. Surv Ophthalmol. 2017;62:838-66.

2. Jia Y, Tan O, Tokayer J, Potsaid B, Wang Y, Liu JJ, et al. Splitspectrum amplitude-decorrelation angiography with optical coherence tomography. Opt Express. 2012;20:4710-25.

3. De Carlo TE, Romano A, Waheed NK, Duker JS. A review of optical coherence tomography angiography (OCTA). Int J Retina Vitreous. 2015;1:5.

4. Jia Y, Bailey ST, Hwang TS, McClintic SM, Gao SS, Pennesi $\mathrm{ME}$, et al. Quantitative optical coherence tomography angiography of vascular abnormalities in the living human eye. Proc Natl Acad Sci. 2015;112:E2395-402.

5. Akil H, Falavarjani KG, Sadda SR, Sadun AA. Optical coherence tomography angiography of the optic disc; an overview. J Ophthalmic Vis Res. 2017;12:98. 
6. Bazvand F, Mirshahi R, Fadakar K, Faghihi H, Sabour S, Ghassemi $\mathrm{F}$. The quantitative measurements of vascular density and flow area of optic nerve head using optical coherence tomography angiography. J Glaucoma. 2017;26:735-41.

7. Falavarjani KG, Sarraf D. Optical coherence tomography angiography of the retina and choroid; current applications and future directions. J Curr Ophthalmol. 2017;29:1-4.

8. Ghassemi F, Fadakar K, Bazvand F, Mirshahi R, Mohebbi M, Sabour S. The quantitative measurements of vascular density and flow areas of macula using optical coherence tomography angiography in normal volunteers. Ophthalmic Surg Lasers Imaging Retina. 2017;48:478-86.

9. Falavarjani KG, Shenazandi H, Naseri D, Anvari P, Sedaghat A, Hashemi M, et al. Correlation of optic disc morphometry and optic disc microvasculature assessed with optical coherence tomography angiography. Can J Ophthalmol. 2018;53:595-9.

10. Torres VL, Brugnoni N, Kaiser PK, Singh AD. Optical coherence tomography enhanced depth imaging of choroidal tumors. Am J Ophthalmol. 2011;151:586-93.e2.

11. Shields CL, Furuta M, Mashayekhi A, Berman EL, Zahler JD, Hoberman DM, et al. Clinical spectrum of choroidal nevi based on age at presentation in 3422 consecutive eyes. Ophthalmology. 2008;115:546-52.e2.

12. Egan KM, Seddon JM, Glynn RJ, Gragoudas ES, Albert DM. Epidemiologic aspects of uveal melanoma. Surv Ophthalmol. 1988;32:239-51.

13. Shields CL, Furuta M, Berman EL, Zahler JD, Hoberman DM, Dinh $\mathrm{DH}$, et al. Choroidal nevus transformation into melanoma: analysis of 2514 consecutive cases. Arch Ophthalmol. 2009;127:981-7.

14. Singh AD, Kalyani P, Topham A. Estimating the risk of malignant transformation of a choroidal nevus. Ophthalmology. 2005; 112:1784-9.

15. Shields JA, Demirci H, Mashayekhi A, Shields CL. Melanocytoma of optic disc in 115 cases: the 2004 Samuel Johnson memorial lecture, part 1. Ophthalmology. 2004;111:1739-46.

16. John D, Earle M, Fine SL, Hawkins BS, Straatsma M, Ocular C. The COMS randomized trial of iodine 125 brachytherapy for choroidal melanoma, III: initial mortality findings. Arch Ophthalmol. 2001;119:969-82.

17. Lee MD, Kaidonis G, Kim AY, Shields RA, Leng T. En face optical coherence tomography angiography imaging versus fundus photography in the measurement of choroidal nevi. Ophthalmic Surg Lasers Imaging Retina. 2017;48:741-7.

18. Ali ZC, Gray J, Balaskas K. Features of choroidal naevi on swept source optical coherence tomography angiography and structural reverse flow optical coherence tomography. Graefes Arch Clin Exp Ophthalmol. 2018;256:1319-23.

19. Shields CL, Kaliki S, Rojanaporn D, Ferenczy SR, Shields JA. Enhanced depth imaging optical coherence tomography of small choroidal melanoma: comparison with choroidal nevus. Arch Ophthalmol. 2012;130:850-6.

20. Folberg R, Mehaffey M, Gardner LM, Meyer M, Rummelt V, Pe'er J. The microcirculation of choroidal and ciliary body melanomas. Eye. 1997;11:227-38.

21. Mueller AJ, Bartsch D-U, Folberg R, Mehaffey MG, Boldt HC, Meyer M, et al. Imaging the microvasculature of choroidal melanomas with confocal indocyanine green scanning laser ophthalmoscopy. Arch Ophthalmol. 1998;116:31-9.

22. Edwards WC, Layden WE, Macdonald R. Fluorescein angiography of malignant melanoma of the choroid. Am J Ophthalmol. 1969;68:797-808.

23. Maloca P, Gyger C, Hasler PW. A pilot study to image the vascular network of small melanocytic choroidal tumors with speckle noise-free 1050-nm swept source optical coherence tomography (OCT choroidal angiography). Graefes Arch Clin Exp Ophthalmol. 2016;254:1201-10.
24. Toledo JJ, Asencio M, García JR, Morales LA, Tomkinson C, Cajigal C. OCT angiography: imaging of choroidal and retinal tumors. Ophthalmol Retina. 2018;2:613-22.

25. Ghassemi F, Mirshahi R, Fadakar K, Sabour S. Optical coherence tomography angiography in choroidal melanoma and nevus. Clin Ophthalmol. 2018;12:207-14.

26. Garcia-Arumi Fuste C, Peralta Iturburu F, Garcia-Arumi J. Is optical coherence tomography angiography helpful in the differential diagnosis of choroidal nevus versus melanoma? Eur J Ophthalmol. 2019. https://doi.org/10.1177/1120672119851768. E-pub ahead of print.

27. Cennamo G, Romano MR, Breve MA, Velotti N, Reibaldi M, de Crecchio G. et al. Evaluation of choroidal tumors with optical coherence tomography: enhanced depth imaging and OCTangiography features. Eye. 2017;31:906-15.

28. Pellegrini M, Staurenghi G. Swept-source optical coherence tomography angiography imaging in a case of uveal melanoma. Ophthalmology. 2017;124:729.

29. Pellegrini M, Corvi F, Invernizzi A, Ravera V, Cereda MG, Staurenghi G. Swept-source optical coherence tomography angiography in choroidal melanoma: an analysis of 22 consecutive cases. Retina. 2018;39:1510-9.

30. Lumbroso B, Huang D, Chen CJ, Jia Y, Rispoli M, Romano A, et al. Clinical OCT angiography atlas. JP Medical Ltd: New Delhi, India, 2015.

31. Li Y, Say EA, Ferenczy S, Agni M, Shields CL. Altered parafoveal microvasculature in treatment-naive choroidal melanoma eyes detected by optical coherence tomography angiography. Retina. 2017;37:32-40.

32. Valverde-Megias A, Say EA, Ferenczy SR, Shields CL. Differential macular features on optical coherence tomography angiography in eyes with choroidal nevus and melanoma. Retina. 2017;37:731-40.

33. Cicinelli MV, Iuliano L, Rabiolo A, Marchese A, Querques G, Bandello F. Optical coherence tomography angiography of polypoidal neovascularization associated with choroidal nevus. Eur J Ophthalmol. 2017;27:9-12.

34. Pellegrini M, Corvi F, Say EAT, Shields CL, Staurenghi G. Optical coherence tomography angiography features of choroidal neovascularization associated with choroidal nevus. Retina. 2018;38:1338-46.

35. Kikuchi I, Kase S, Hashimoto Y, Hirooka K, Ishida S. Involvement of circulatory disturbance in optic disk melanocytoma with visual dysfunction. Graefes Arch Clin Exp Ophthalmol. 2019;257:835-41.

36. Naseripour M, Jaberi R, Sedaghat A, Azma Z, Nojomi M, Falavarjani KG, et al. Ruthenium-106 brachytherapy for thick uveal melanoma: reappraisal of apex and base dose radiation and dose rate. J Contemp Brachytherapy. 2016;8:66-73.

37. Cennamo G, Breve MA, Velotti N, Sparnelli F, Iovino C, Farella A, et al. Evaluation of vascular changes with optical coherence tomography angiography after plaque radiotherapy of choroidal melanoma. Ophthalmic Res. 2018;60:238-42.

38. Veverka KK, AbouChehade JE, Iezzi R Jr., Pulido JS. Noninvasive grading of radiation retinopathy: the use of optical coherence tomography angiography. Retina. 2015;35:2400-10.

39. Horgan N, Shields CL, Mashayekhi A, Teixeira LF, Materin MA, Shields JA. Early macular morphological changes following plaque radiotherapy for uveal melanoma. Retina. 2008;28:263-73.

40. Matet A, Daruich A, Zografos L. Radiation maculopathy after proton beam therapy for uveal melanoma: optical coherence tomography angiography alterations influencing visual acuity. Invest Ophthalmol Vis Sci. 2017;58:3851-61.

41. Shields CL, Say EA, Samara WA, Khoo CT, Mashayekhi A, Shields JA. Optical coherence tomography angiography of the 
macula after plaque radiotherapy of choroidal melanoma: comparison of irradiated versus nonirradiated eyes in 65 patients. Retina. 2016;36:1493-505.

42. Say EA, Samara WA, Khoo CT, Magrath GN, Sharma P, Ferenczy $\mathrm{S}$, et al. Parafoveal capillary density after plaque radiotherapy for choroidal melanoma: analysis of eyes without radiation maculopathy. Retina. 2016;36:1670-8.

43. Sellam A, Coscas F, Lumbroso-Le Rouic L, Dendale R, Lupidi M, Coscas G, et al. Optical coherence tomography angiography of macular features after proton beam radiotherapy for small choroidal melanoma. Am J Ophthalmol. 2017;181:12-9.

44. Naseripour M, Singh AD. Uveal vascular tumors. In: Clinical ophthalmic oncology. Springer, Berlin, 2014, pp 287-306.

45. Takkar B, Azad S, Shakrawal J, Gaur N, Venkatesh P. Blood flow pattern in a choroidal hemangioma imaged on swept-sourceoptical coherence tomography angiography. Indian J Ophthalmol. 2017;65:1240-2.

46. Konana VK, Shanmugam PM, Ramanjulu R, Mishra KCD, Sagar P. Optical coherence tomography angiography features of choroidal hemangioma. Indian J Ophthalmol. 2018;66:581-3.

47. Chen FK, Viljoen RD, Bukowska DM. Classification of image artefacts in optical coherence tomography angiography of the choroid in macular diseases. Clin Exp Ophthalmol. 2016;44:388-99.

48. Sweeney AR, Zhang Q, Wang RK, Rezaei KA. Optical coherence tomography microangiography imaging of circumscribed choroidal hemangioma. Ophthalmic Surg Lasers Imaging Retina. 2018;49:134-7.

49. Naseripour M, Maleki A, Astaraki A, Sedaghat A, Jaberi R, Lee S, et al. Ruthenium-106 brachytherapy in the treatment of circumscribed choroidal hemangioma. Retina. 2018;38:1024-30.

50. Chawla R, Tripathy K, Sharma A, Vohra R. Swept source optical coherence tomography-angiography of choroid in choroidal hemangioma before and after laser photocoagulation. Indian $\mathbf{J}$ Ophthalmol. 2017;65:751-4.

51. Gass JDM. Stereoscopic atlas of macular diseases: diagnosis and treatment (2 Volume Set). St. Louis: Mosby; 1997.

52. Giudice GL, Galan A. Optical coherence tomography angiography of circumscribed choroidal hemangioma treated with photodynamic therapy. Indian J Ophthalmol. 2017;65:1049-51.

53. Cennamo G, Rossi C, Breve MA, Velotti N, Farella A, Liuzzi R, et al. Evaluation of vascular changes with optical coherence tomography angiography after ruthenium-106 brachytherapy of circumscribed choroidal hemangioma. Eye. 2018;32:1401-5.

54. Gass JDM, Guerry RK, Jack RL, Harris G. Choroidal osteoma. Arch Ophthalmol. 1978;96:428-35.

55. Shields CL, Shields JA, Augsburger JJ. Choroidal osteoma. Surv Ophthalmol. 1988;33:17-27.

56. Trimble SN, Schatz H, Schneider GB. Spontaneous decalcification of a choroidal osteoma. Ophthalmology. 1988;95:631-4.

57. Shields CL, Sun H, Demirci H, Shields JA. Factors predictive of tumor growth, tumor decalcification, choroidal neovascularization, and visual outcome in 74 eyes with choroidal osteoma. Arch Ophthalmol. 2005;123:1658-66.

58. Shen C, Yan S, Du M, Zhao H, Shao L, Hu Y. Assessment of choroidal osteoma complicating choroidal neovascularization by optical coherence tomography angiography. Int Ophthalmol. 2018;38:787-92.
59. Azad SV, Takkar B, Venkatesh P, Kumar A. Swept source: optical coherence tomography angiography features of choroidal osteoma with choroidal neovascular membrane. BMJ Case Rep. 2016;2016:bcr2016215899.

60. Szelog JT, Bonini Filho MA, Lally DR, de Carlo TE, Duker JS. Optical coherence tomography angiography for detecting choroidal neovascularization secondary to choroidal osteoma. Ophthalmic Surg Lasers Imaging Retina. 2016;47:69-72.

61. Furino C, Antonio LD, Grassi MO, Rispoli M, Reibaldi M, Niro A, et al. Choroidal neovascularization due to choroidal osteoma treated with anti-vascular endothelial growth factor therapy: an optical coherence tomography angiography study. Eur J Ophthalmol. 2019;29:323-29.

62. Mathis T, Jardel P, Loria O, Delaunay B, Nguyen AM, Lanza F, et al. New concepts in the diagnosis and management of choroidal metastases. Prog Retina Eye Res. 2018;68:144-76.

63. Sioufi K, Say EAT, Ferenczy SC, Leahey AM, Shields CL. Optical coherence tomography angiography findings of deep capillary plexus microischemia after intravenous chemotherapy for retinoblastoma. Retina. 2019;39:371-78.

64. Arrigo A, Corbelli E, Aragona E, Manitto MP, Martina E, Bandello F, et al. Optical coherence tomography and optical coherence tomography angiography evaluation of combined hamartoma of the retina and retinal pigment epithelium. Retina. 2019;39:1009-15.

65. Hoobyar AR, Ferrucci S, Anderson SF, Townsend JC. Juxtapapillary capillary hemangioblastoma. Optom Vis Sci. 2002;79:346-52.

66. Thirumalesh MB, Jain A, Agrawal S, Bhujang Shetty K. In vivo microvascular pattern of solitary juxtapapillary capillary hemangioma on OCT angiography. Ophthalmic Surg Lasers Imaging Retina. 2017;48:592-5.

67. Ferenczy SR, Shields CL. Optical coherence tomography angiography: an overview of applications in ocular oncology. J Ophthalmic Photogr. 2018;40:70-8.

68. Kang AS, Welch RJ, Sioufi K, Say EAT, Shields JA, Shields CL. Optical coherence tomography angiography of iris microhemangiomatosis. Am J Ophthalmol Case Rep. 2017;6:24-6.

69. Chien JL, Sioufi K, Ferenczy S, Say EAT, Shields CL. Optical coherence tomography angiography features of iris racemose hemangioma in 4 cases. JAMA Ophthalmol. 2017;135:1106-10.

70. Skalet AH, Li Y, Lu CD, Jia Y, Lee B, Husvogt L, et al. Optical coherence tomography angiography characteristics of iris melanocytic tumors. Ophthalmology. 2017;124:197-204.

71. Spaide RF, Fujimoto JG, Waheed NK. Image artifacts in optical coherence angiography. Retina. 2015;35:2163-80.

72. Falavarjani KG, Al-Sheikh M, Akil H, Sadda SR. Image artefacts in swept-source optical coherence tomography angiography. Br J Ophthalmol. 2017;101:564-8.

73. Say EAT, Ferenczy S, Magrath GN, Samara WA, Khoo CTL, Shields CL. Image quality and artifacts on optical coherence tomography angiography: comparison of pathologic and paired fellow eyes in 65 patients with unilateral choroidal melanoma treated with plaque radiotherapy. Retina. 2017;37:1660-73.

74. Al-Sheikh M, Falavarjani KG, Akil H, Sadda SR. Impact of image quality on OCT angiography based quantitative measurements. Int J Retina Vitreous. 2017;3:13. 